\title{
Microchip Site
}

National Cancer Institute

\section{Source}

National Cancer Institute. Microchip Site. NCI Thesaurus. Code C77682.

The anatomic site at which a microchip is implanted. 